\title{
En qué cambian los pacientes drogodependientes a los seis meses de tratamiento en la comunidad terapéutica.
}

\author{
How patients who are dependent on drugs change after six months' treatment in the thera- \\ peutic community.
}

\section{Fernando Pérez del Río ${ }^{\text {a }}$}

${ }^{a}$ Doctor en Psicología. Proyecto Hombre Burgos, España.

Correspondencia: Fernando Pérezdel Río (fernando@proyectohombreburgos.com)

Recibido: 11/08/2011; aceptado con modificaciones: 17/11/2011

RESUMEN: Introducción. El objetivo de este estudio es evidenciar en qué cambian los pacientes drogodependientes a los seis meses de tratamiento en la Comunidad Terapéutica (CT).

Material y método. Se aplicaron ocho pruebas diagnosticas al primer mes y a los seis meses de tratamiento, diseño pre test y post test. Los cuestionarios fueron:

EuropASI. Cuestionario de Psicopatología (SCL90-R). Cuestionario de Agresión (AQ). Trastornos de Personalidad (IPDE). Cuestionario de Autoeficacia (SES). Escala de Evaluación del Cambio (URICA). Inventario de Procesos de Cambio (PCQ). Cuestionario de Balance Decisional (DBC). Se evalúa a 159 pacientes ingresados en Comunidad Terapéutica diagnosticados de dependencia a sustancias según el DSM IV-TR.

Para realizar el perfil sociodemográfico y de gravedad de la adicción se efectuó un análisis de Conglomerados en dos Fases de los datos derivados del EuropASI. La edad media de los participantes es de 33 años (rango: 22-56 años). Para los restantes cuestionarios, se tipificaron en puntuaciones $\mathrm{Z}$ las variables dependientes y después se realizaron análisis de varianzas con medidas repetidas (ANOVA). Resultados y conclusiones. Se cumplen los requisitos para sostener que se dan cambios significativos en las siguientes variables estudiadas: a) no consumen sustancias; b) avanzan a la fase de Acción; c) se dan cambios en el Balance Decisional; d) aumentan en Autoeficacia; e) se dan cambios en la variable Hostilidad; f) aumentan en cinco Procesos de Cambio.

PALABRAS CLAVE: Drogodependientes. Adictos. Tratamientos. Comunidad Terapéutica. Evaluación.
ABSTRACT: Introduction. The aim of this study is to show how patients who are dependent on drugs change after six months' treatment in the Therapeutic Community (TC).

Material and methods. Eight diagnostic tests were performed after the first month and after six months' treatment, pre test and post test design. The questionnaires used were:

EuropASI. Psychopathology Questionnaire (SCL90-R). Aggression Questionnaire (AQ). Personality Disorders (IPDE). Self-Efficacy Questionnaire (SES).Change Assessment Scale (URICA). Process of Change Questionnaire (PCQ). Decisional Balance Questionnaire (DBC).

76 patients in the TC diagnosed as dependent on substances were assessed according to DSM IV-TR.

In order to carry out the socio-demographic profile and the seriousness to addiction profile, it was accomplished an analysis of Conglomerate in two phases with the data resulted in EuropASI. The average age of the participants was 33 years (range: $22-56$ years).

For the rest of the questionnaires, the dependent variables were classified in Z-scores and analyses of variance were then performed with repeated averages (ANOVA).

Results and conclusions. The requirements were met for maintaining that there are significant changes in the following variables that were studied: (a) they do not consume substances; (b) they progress to the Action phase; (c) there are changes in the Decisional Balance; (d) their Self-Efficacy increases; (e) there are changes to the Hostility variable; (f) there are increases in five Change Processes.

KEY WORDS: Drug Addicts. Addicts. Treatments. Therapeutic Community. Assessment. 
ORIGINALES Y REVISIONES

\section{Introducción}

Los estudios de seguimiento han sido frecuentes para evidenciar la eficacia de los tratamientos y el cambio de los pacientes drogodependientes. Los países anglosajones son los que más tradición atesoran a la hora de implantar indicadores de referencia con el fin de evaluar. Por ejemplo es ya clásica la evaluación del tratamiento realizada en Inglaterra denominada N.T.O.R.S.; y en Escocia encontramos el Project Drug Outcome Research in Scotland study (D.O.R.I.S.). En EE.UU., periódicamente se realizan macro-estudios de evaluación de tratamientos, por ejemplo el Drug Abuse Reporting Program (D.A.R.P.), el Treatment Outcome Prospective Study (T.O.P.S.) y, más recientemente, el Drug Abuse Treatment Outcome Study (D.A.T.O.S.) (1).

En cuanto a España, los primeros estudios de seguimiento fueron trabajos básicamente de verificación, pues pretendían confirmar estadísticamente los perfiles de drogodependientes. Los primeros estudios "pioneros" fueron con heroinómanos (Bedate y Romero, 1985; Melgajero y col., 1986; Sánchez-Carbonell, Brigos, y Camí, 1989) (2.3.4.). En líneas generales podemos diferenciar dos períodos: antes de la creación del Plan Nacional sobre Drogas (PNsD) en 1985 y después. Antes los estudios partían de centros privados y ONGs de forma bastante aislada, eran trabajos descriptivos, sociodemográficos y algunos -los menos- de seguimiento. En España en un segundo periodo el esfuerzo de los estudios, que siguen siendo infrecuentes, ha sido destinado sobre todo a evidenciar la eficacia del tratamiento como consecuencia de la necesidad de consolidar los programas asistenciales para drogodependientes creados sobre todo en las décadas de los 80 y 90.

Podemos destacar entre otros el estudio comparativo de la eficacia de los programas de tratamiento de Proyecto Hombre de Asturias, Galicia y Baleares, realizado por Fernández y Secades 2001 (5), o recientes trabajos como el de Goñi 2005 en Pamplona (6) y el estudio de seguimiento realizado en la Comunidad Terapéutica Haize-Gain en San Sebastián, García, Roldán, Comas (2010) (7).

El presente estudio de seguimiento se ha centrado en un objetivo un tanto diferente y no es otro que mostrar en qué variables psicológicas cambian los pacientes en un periodo corto de tiempo. Se analizó cómo estaban los pacientes en el primer mes y qué cambios positivos se dieron al medio año de tratamiento en relación a las variables registradas.

En primer lugar se analizan las características de la muestra para evidenciar los posibles perfiles de los usuarios.

Tras esto se analizan las diferencias existentes en una serie de variables, en primer lugar los llamados: a) procesos de cambio (aumento de la concienciación, relieve dramático, contracondicionamiento, liberación social, reevaluación ambiental, manejo de contingencias, autoliberación, relaciones de ayuda y control 
ORIGINALES Y REVISIONES

de estímulos); b) sin cambiar de escenario, también se mide y compara el balance decisional, que son los pros y contras de consumir sustancias psicoactivas; c) en tercer lugar se coteja el cambio a los seis meses de las expectativas de autoeficacia (tentación de consumir y confianza en no consumir); d) en cuarto lugar se mide la variable agresión en cuatro formas (agresión física, agresión psicológica, hostilidad e irascibilidad); e) y por último se aplica un cuestionario de evaluación del cambio donde se advierte en qué etapa de cambio están los usuarios en el momento de la evaluación.

\section{Material y método}

La Comunidad Terapéutica El Priorato, está ubicada en San Medel (Burgos). Es un centro residencial que da cabida a una serie de servicios que facilitan el cambio y la mejora de la calidad de vida de las personas con problemas de adicciones. Los tratamientos son individualizados, se trabaja por objetivos acordados con el paciente. Un equipo multidisciplinar desarrolla terapia individual, grupal, familiar y se imparten diferentes talleres educativos. El abordaje del tratamiento de las adicciones es biopsicosocial y fundamentalemnte psico-educativo. La CT se divide en tres fases que son flexibles, la primera es una etapa de desintoxicación y de adaptación al centro, la segunda es donde se suele ahondar en un mayor trabajo terapéutico educativo y, en la tercera, se da mayor importancia a lo socio-laboral. El centro posee la ISO 9001:2008 UNE desde 2004.

En cuanto a la obtención de los participantes, se seleccionaron todos los usuarios mayores de 21 años que ingresaron consecutivamente en la CT en un arco temporal desde junio de 2005 hasta enero de 2008. La colaboración fue voluntaria y ninguno rechazó participar en el estudio, todos firmaron un documento de autorización cumpliendo lo dispuesto en el artículo 12 de la Ley Orgánica 15/1999, del 13 de diciembre, de Protección de Datos de Carácter Personal. Todas las personas fueron diagnosticadas de dependencia a sustancias según los criterios del DSM IV$T R$ (8). 156 usuarios realizaron la primera evaluación y 76 participantes llegaron a realizar la segunda evaluación en la CT a los seis meses. La edad media de los participantes es de 33 años (rango: 22-56 años). En la primera fase los instrumentos de evaluación aplicados fueron los siguientes:

- EuroASI (Addiction Severity Index) (9). Este cuestionario recoge datos sociodemográficos, situación médica, empleo/soportes, uso de alcohol y drogas, situación legal, historia familiar, relaciones familiares y sociales, y estado psiquiátrico.

- SCL-90-R (10). Baltimore Clinical Psychometric Research, es un Inventario 
de Evaluación Clínica de síntomas de Psicopatología. Se trata de un cuestionario autoaplicable que consta de 90 síntomas. Tiene cinco niveles de respuesta (0-4).

- Examen Internacional de Trastornos de la Personalidad (IPDE) (11). El cuestionario autoaplicado consta de 59 ítems dicotómicos, verdadero vs falso. El cuestionario (screening) subraya e identifica pautas en relación a los trastornos de personalidad: F60.0 Paranoide, F60.1 Esquizoide, F60.2 Disocial, F60.30 Impulsivo, F60.31 Límite, F60.4 Histriónico, F60.5 Anancástico, F60.6 Ansioso, F60.7 Dependiente.

- Escala de Evaluación del Cambio de la Universidad de Rhode Island (URI$C A)$ (12). Es un autoinforme destinado a evaluar el estado de cambio respecto a la modificación de cualquier conducta problema. Esta escala evalúa cuatro de los cinco estadios de cambio diseñados según el modelo Transteórico de Prochaska y DiClemente: precontemplación, contemplación, acción, mantenimiento (13).

- Inventario de Procesos de Cambio (IPC) (14), versión genérica: drogas. El objetivo de este inventario es tratar de identificar qué procesos de cambio (estrategias cognitivo conductuales) ha desarrollado un determinado paciente según el modelo Transteórico (15). Los procesos de cambio son los siguientes:

Aumento de la concienciación: consiste en aumentar la cantidad de información sobre uno mismo, desarrollar el ser consciente de lo que le ocurre, de los problemas y oportunidades. Prochaska y col (1994) (16) consideran que es el proceso más ampliamente utilizado. "Su descripción inicial parte de la obra de Sigmund Freud, que planteaba que el objetivo del psicoanálisis era hacer consciente lo inconsciente". Autoliberación: es una categoría que nos viene a decir cómo se compromete la persona ante las elecciones que va haciendo. Liberación social: consiste en la liberación emocional de lo que se dice (compartir con otras personas lo que está realizando). Darse permisos para el cambio y después del permiso, procurar formas de actuar nuevas o alternativas frente a las secuencias problemáticas que se vienen sucediendo. Autorreevaluación: la persona con problemas de adicción realiza una evaluación de sí misma (cognitivo afectiva), de su vida, de su problema, y analiza qué consecuencias e impacto sobre su estilo de vida, sus valores, etc., tendría modificar el problema. Reevaluación ambiental: la persona con un problema de adicción realiza una evaluación de la situación, de sus relaciones, y valora las consecuencias de seguir con la conducta adictiva y el beneficio de cambiarlo en torno a sus relaciones interpersonales, familiares, personas allegadas. Contracondicionamiento: se trata de generar respuestas alternativas (tanto a nivel cognitivo, afectivo o conductual) a los estímulos condicionados generados por las drogas y por la conducta adictiva. $\mathrm{O}$ dicho de una forma más simple se trata de procurar actividades que sean alternativas conductuales a la conducta problema. Control de estímulos: es modificar o controlar el ambiente para no encontrarse frente a situaciones de riesgo. Manejo de contingencias: son estrategias conductuales que ayudan a que se manten- 
ORIGINALES Y REVISIONES

ga o aumente una determinada conducta. En este apartado el paciente analiza cómo se refuerzan o como se castigan las conductas. Relieve dramático: consiste en saber y poder expresar lo que se siente, por ejemplo expresar lo emocionalmente negativo asociado a la conducta adictiva. Relaciones de ayuda: consiste en todo el abanico de posibles relaciones personales que pueda ayudar a la persona con problemas de drogas a la hora de emprender su proceso. Mide la existencia de apoyos sociales (familiares, amigos) que pueden facilitar el cambio.

- Cuestionario de Balance Decisional (CBD). Es un autoinforme que fue diseñado con el objetivo de evaluar el "posible conflicto" ante el abandono de la adicción a los opiáceos. En el presente trabajo se utiliza el Cuestionario de Balance Decisional (BD) de Tejero, Trujols y Roca (1994) (17) en su versión genérica: drogas, y no sólo para los dependientes a la heroína. Se pregunta al paciente por los pros y contras del consumo de la sustancia con la que tiene problemas. La subescala de los pros consta de diez ítems que recogen lo positivo de las drogas o de su consumo. La segunda subescala de cuestionario $(C B D)$ consta de otros diez ítems que plantean las consecuencias indeseables y perjudiciales del consumo de drogas.

- Cuestionario de Autoeficacia (Tentación Autoeficacia) (18). Este cuestionario plantea una serie de situaciones de riesgo de consumo y las respuestas ante tales situaciones.

- Cuestionario de Agresión (AQ) (19). Este autoinforme ha sido avalado para medir la agresión en cuatro subescalas denominadas: a) agresividad verbal; b) agresividad física; c) la ira; d) la hostilidad.

La segunda fase de aplicación de cuestionarios se establece cuando el usuario lleva seis meses de tratamiento en la CT. En esta segunda fase se aplican seis protocolos de evaluación, dos de los anteriores cuestionarios, el $S C L-90$ y el cuestionario IPDE, no se emplean en esta segunda vuelta. En relación a estos dos cuestionarios $S C L-90$ e IPDE aplicados en la primera evaluación subrayamos que estamos ante una muestra con más sintomatología psiquiátrica y con significativamente más trastornos de personalidad que la media poblacional. Así lo reflejan las evidencias encontradas en los cuestionarios. Pero se decidió omitir estos dos cuestionarios en la evaluación a los seis meses puesto que se alejaba del objetivo principal del estudio, por otro lado es un tema que sí ha sido más estudiado, por ejemplo el reciente libro Características psicopatológicas, motivacionales y de personalidad en drogodependencias en tratamiento de la Comunidad de Madrid (20) recoge diferentes estudios en esta línea.

Asimismo debemos tener en cuenta la variable "consumos de sustancias psicoactivas," ya que también se registró si los pacientes evaluados consumían o no en el momento de la evaluación.

Después de recuperar todos los cuestionarios correctamente rellenados por los pacientes y tras haberlos corregido, se procedió a introducir los datos en el pro- 
grama informático SPSS con el que se realizaron todos los análisis estadísticos. Las diferentes variables dependientes son las respuestas emitidas por los participantes en los diversos cuestionarios, balance decisional, procesos de cambio, expectativas de autoeficacia, evaluación del cambio y agresión. Se realizan los siguientes análisis: se tipificaron en puntuaciones $\mathrm{Z}$ las variables dependientes, después se realizaron algunos análisis de varianzas con medidas repetidas (ANOVA).

Para obtener un perfil de los usuarios, como ya se indicó, se utilizó el EuropASI y el análisis que se realizó fue: 1) Clasificación de los sujetos mediante un análisis de conglomerados (cluster) en dos fases. 2) Diversas pruebas de ChiCuadrado $(\chi 2)$ de Pearson y $\chi 2$ de la razón de verosimilitud. 3) Pruebas t de Student para muestras independientes. Se tuvo en cuenta la prueba de Levene $(F)$ sobre homogeneidad o igualdad de varianzas. 4) Tipificación (puntuaciones Z) para elaborar un perfil gráfico de los conglomerados.

\section{Resultados}

En cuanto a la muestra de 159 personas con problemas de drogas, el 3,9\% son de nacionalidad extranjera, el 72,5\% de los encuestados viene sin haber estado el último mes en otro tratamiento, el $88,7 \%$ son varones y el $11,3 \%$ mujeres.

En relación a los perfiles

En primer lugar se han obtenido dos grupos bien diferenciados, por un lado los politoxicómanos $(\mathrm{P})$ que fundamentalmente consumen estimulantes como la cocaína, y por otro, los alcohólicos (A) por otro lado. Exponemos un resumen de las características de los dos perfiles encontrados.

Tipo (P) Politoxicómanos.

La media de edad es de 30,87 años, obtienen el dinero para drogas fundamentalmente de su trabajo, de la prestación por desempleo, de actividades ilegales, de los compañeros y de la familia. El grupo $(\mathrm{P})$ atesora más problemas judiciales y se comprende entonces que más admisiones a tratamiento hayan sido promovidas o sugeridas por el sistema judicial.

En el grupo $(\mathrm{P})$ hay más gente soltera $\mathrm{y}$, en cuanto a su familia, gozan de mejores relaciones con los padres y hermanos que el grupo (A) $(p<0.001)$. El grupo $(\mathrm{P})$ consume más psicoestimulantes como la cocaína, pero al mismo tiempo consume otras sustancias; ellos mismos se identifican como "politoxicómanos". La cocaína y las anfetaminas son consumidas durante más años y se comienza a una edad menor su consumo. En general las evidencias indican que hay más familiares con antecedentes por consumo de drogas. 


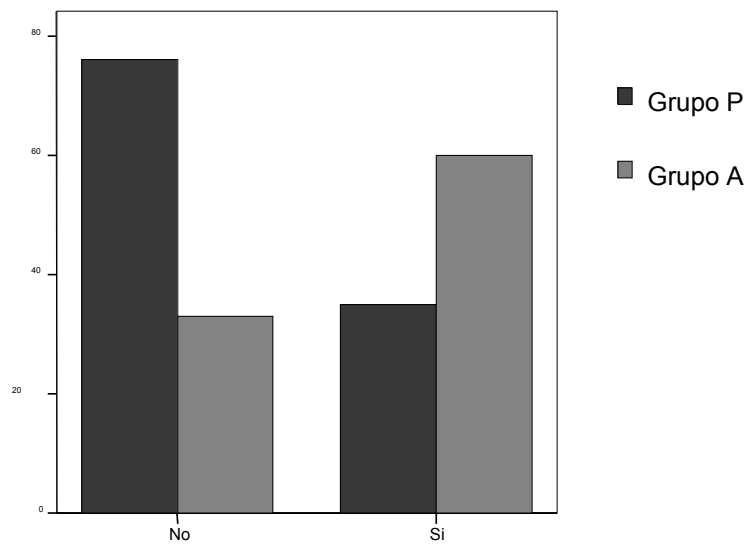

\section{Tipo (A) Alcohólicos}

La media de edad es de 35,72 años. Obtienen más dinero del paro, de las ayudas sociales, de la pensión o la Seguridad Social. Han pasado más tiempo desempleadas y tienen menos deudas en comparación con el anterior grupo $(p<0.001)$. Hay más antecedentes familiares (padres, tíos, etc.) con un historial de consumo de alcohol. Encontramos más personas divorciadas o separadas. Hallamos más usuarios de tipo "errantes", es decir que no tienen residencia fija. Un mayor número de pacientes de este grupo viven solos. Los miembros del grupo atestiguan haber tenido peores relaciones con los hermanos, con la pareja, con la madre y padre, con todos salvo con los que no son familiares.

Este grupo ha consumido de media 9,61 años (grandes cantidades de alcohol), antes de entrar en tratamiento.

Para el grupo (A) tratar los problemas relacionados con el alcohol es extremadamente importante. Su situación médica es calificada igualmente como grave y también afirman que la gravedad de sus problemas familiares y sociales es extrema, y que el tratamiento en estas áreas es absolutamente necesario. El grupo de alcohol se percibe con más problemas psicológicos. Y reciben más medicación prescrita por problemas emocionales o psicológicos $(p<0.001)$ en comparación con el grupo (P). Más participantes del grupo de alcohol han padecido depresión severa e intentos de suicidio, experimentaron más alucinaciones y han pasado más tiempo hospitalizados. Tuvieron significativamente más problemas para comprender 0 concentrarse. Podemos destacar que los participantes de esta tipología (A) que llegan a pedir tratamiento están más deteriorados en todo lo referente a lo psiquiátrico que el grupo $(\mathrm{P})(p<0.001)$. 


\section{Variables de buen pronóstico}

En otro orden de cosas, encontramos algunas diferencias significativas entre los pacientes que realizan seis meses de tratamiento en la Comunidad Terapéutica en comparación con los que abandonan: a) los pacientes que llegan a los seis meses están ligeramente menos deteriorados que los que abandonan el recurso; b) han consumido menos drogas y durante menos años; c) han pasado periodos más largos abstinentes de drogas o alcohol; d) comienzan a consumir alcohol o drogas a una edad más tardía. Estas diferencias se encontraron tanto en el grupo (A) y (P).

En cualquier caso, parece recomendable evitar la simplificación y calificar de "peor pronóstico" a los pacientes del grupo que abandonan el recurso, por lo que supone de repercusión y estigmatización en la práctica diaria. Este "peor pronóstico" es preferible matizarlo y decir que son personas que necesitan por ejemplo, cuidados especiales.

Por último, en relación al número de personas que abandonaron y no llegaron a realizar la evaluación a los seis meses, hay que apuntar que hubo muchas dificultades en su contabilización puesto que una misma persona podía entrar y salir hasta en tres o cuatro ocasiones de la CT. Pese a este aprieto el porcentaje de abandonos por muy diferentes motivos fue de 83 , estaría en torno al $50 \%$ en los primeros seis meses, siendo similar a otras CTs. También hay que recordar que la mayor parte de los abandonos en todo el transcurso del programa se producen en el primer y tercer mes tras el ingreso.

En relación a la variable consumo de sustancias, ninguno de los 76 entrevistados consumía sustancias en el segundo momento de la evaluación (a los seis meses).

a) Cuestionario Balance Decisional $(C B D)$, en cuanto a los pros $[\mathrm{F}(1,75)=$ $118, \mathrm{p} \leq 0,001]$ vemos que hay una disminución a los seis meses de tratamiento, asimismo se evidencia que a los seis meses de tratamiento también hay menos contras, $\left[\mathrm{F}_{(1,75)}=2569, \mathrm{p} \leq 0,001\right]$.

Gráfico 2:

Cuestionario Balance Decisional: Pros

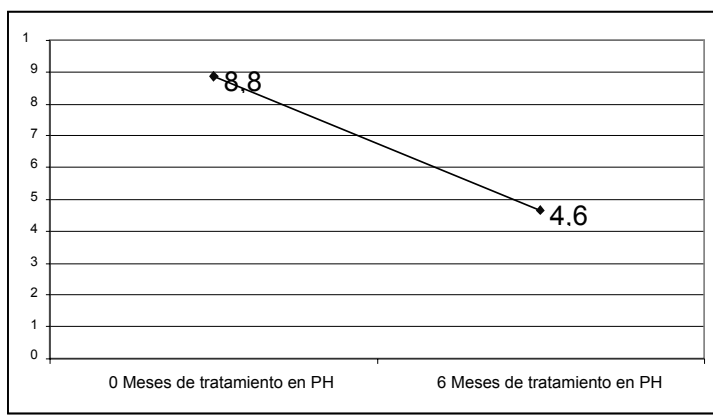


Gráfico 3.

Cuestionario Balance Decisional: Contras

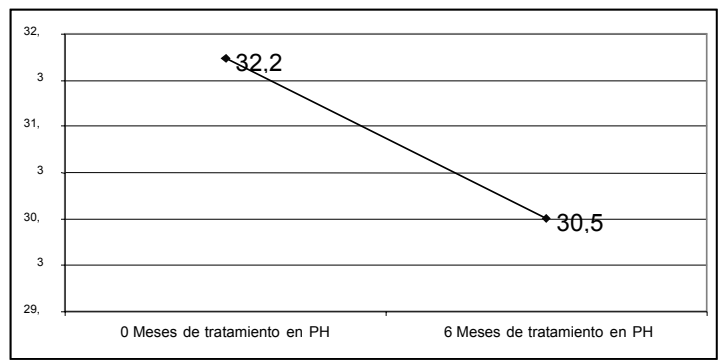

b) Cuestionario de Autoeficacia (Tentación y Confianza) (SES). Las puntuaciones en la variable tentación de consumo de sustancias son significativas $[\mathrm{F}(1,72)$ $=32, \mathrm{p} \leq 0,001]$. Lo mismo ocurre con la confianza que a los seis meses es mucho $\operatorname{mayor}\left[\mathrm{F}_{(1,73)}=1440, \mathrm{p} \leq 0,001\right]$.

Gráfico 4:

Cuestionario de Autoeficacia. Tentación de consumir drogas

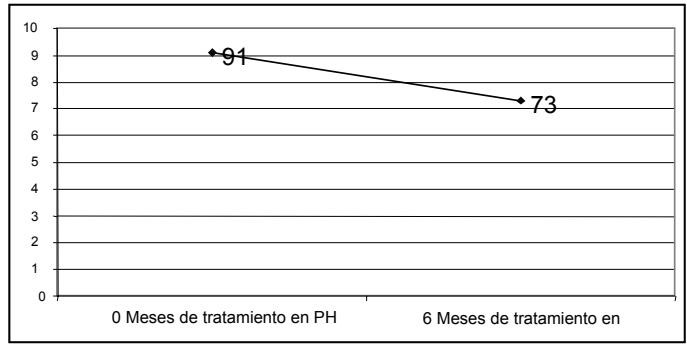

Gráfico 5:

Cuestionario de Autoeficacia. Confianza en no consumir drogas

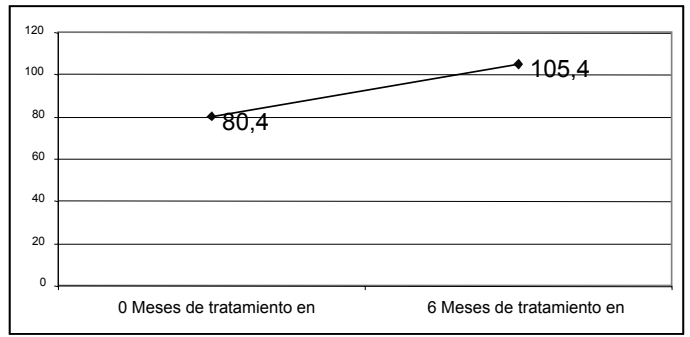


c) Estadios de Evaluación del Cambio (URICA). A los seis meses de tratamiento la fase que aumenta es la etapa de acción $\left[\mathrm{F}_{(1,75)}=11970, \mathrm{p} \leq 0,001\right]$.

d) Inventario de Procesos de Cambio (IPQ). De todos los procesos mencionaremos los significativos. En relación a la variable liberación social encontramos que hay un aumento considerable $[\mathrm{F}(1,75)=2200, \mathrm{p} \leq 0,001]$. A los seis meses la variable re-evaluación ambiental también aumenta $[\mathrm{F}(1,75)=4725, \mathrm{p} \leq 0,001]$. La variable cotracondicionamiento aumenta significativamente, la persona ha comenzado actividades alternativas $[\mathrm{F}(1,75)=388, \mathrm{p} \leq 0,001]$. El control de estímulos es una variable que aumenta su magnitud a los seis meses $[\mathrm{F}(1,75)=384, \mathrm{p} \leq 0,001]$. Por último, en relación a la variable relaciones de ayuda, la diferencia encontrada es significativa estadísticamente $[\mathrm{F}(1,75)=1195, \mathrm{p} \leq 0,001]$.

e) Cuestionario de Agresión ( $A Q$ ). El dato más destacable de todo lo relacionado con la agresividad es el significativo aumento de la hostilidad $\left[\mathrm{F}_{(1,75)}=1461\right.$, $\mathrm{p}<0,001]$.

\section{Discusión}

Antes de nada, resulta necesario revisar la literatura científica de la Comunidad Terapéutica, ya que esta nos subraya determinados aspectos, muchos de ellos en principio obvios; por ejemplo, que es mejor seguir un tratamiento de rehabilitación que no seguir ningún tratamiento o no hacer nada, o que "existe relación entre acudir a grupos de terapia y mejorar" (21).

En cuanto a la variable de consumo, a los seis meses ninguno de los 76 encuestados consume ningún tipo de drogas salvo tabaco. Este hecho se debe, entre otros, a que el paciente está en un entorno controlado donde se realizan analíticas de forma aleatoria de Morfina/Heroína, Cocaína, Benzodiacepinas, y THC.

Otros estudios también han verificado el cese de consumo de drogas. Tras realizar un análisis de los estudios de eficacia en Proyecto Hombre, como conclusión general se puede afirmar que el programa educativo terapéutico de Proyecto Hombre cumple sus objetivos. Los datos de las personas que obtuvieron el alta indican que la mayoría de las mismas están abstinentes del consumo de drogas ilegales y no consumen alcohol o lo hacen de manera moderada (22).

En cuanto al Balance Decisional (CBD), pros y contras, a los seis meses encontramos menos pros y menos contras. Como decíamos, este cuestionario mide el conflicto decisional relacionado con el abandono del consumo de sustancias; al inicio del tratamiento las posturas son más "extremas" y podemos interpretar que a los seis meses las posturas se "suavizan", aunque esta respuesta no aclara del todo este hallazgo. Otra explicación a este hecho es que la terapia se enfoca a las dificultades 
ORIGINALES Y REVISIONES

de la persona y no únicamente al consumo de sustancias psicoactivas, esto sería coherente pues en el tratamiento, ya desde la primera entrevista, se suele recomendar no focalizar el problema únicamente en las drogas (23). Se trabaja con la función del síntoma. "En la linguiística actual, 'síntoma' se utiliza a veces como sinónimo de una de las funciones del lenguaje que Büller, en 1918, denominó Kundgabe o función 'expresiva', esto es, la que permite al hablante expresar su estado psíquico" (24). Expuesto lo anterior, resulta comprensible que los pros y contras de consumir pierdan "y deban perder" importancia frente a las otras muchas áreas que se trabajan con los usuarios. En este sentido, no centrar la terapia exclusivamente en la sustancia se esboza como una variable positiva en el éxito terapéutico.

Otra de las conclusiones más significativas la encontramos en la variable $\mathrm{Au}$ toeficacia (Tentación y Confianza). De una forma muy evidente observamos que la tentación baja muy significativamente y por otro lado la confianza aumenta considerablemente. Esto quiere decir que a los seis meses de tratamiento se amplía significativamente la variable autoeficacia. Es frecuente que cuando un paciente se siente enfadado o deprimido recurra al consumo pero a los seis meses de tratamiento esto cambiaría.

En relación al tratamiento encontramos estudios que nos muestran que "las personas que siguen terapia para dejar la adicción mejoran sus habilidades de afrontamiento y autoeficacia" (25). Sin duda la autoeficacia ha mostrado ser un constructo de elevada utilidad clínica en las drogodependencias $(26,27)$. También se ha encontrado una relación significativa entre el aumento de autoeficacia y el mantenimiento exitoso del abandono del tabaco (28). Dada la literatura revisada se concluye que una mayor autoeficacia es un buen indicador de menores consumos de sustancias y de menores futuras recaídas. "La expectativa de autoeficacia permanece baja en la precontemplación y contemplación, y aumenta conforme el drogodependiente pasa hacia el estadio de acción y alcanzan su máxima expresión en la etapa de mantenimiento" (29). Sin duda la autoeficacia es una variable que resulta determinante en situaciones complicadas.

URICA. Otros resultados obtenidos derivados del Cuestionario Estadios de Evaluación del Cambio (URICA) evidencian que a los seis meses la única fase que aumenta es la fase de acción. Es fácil encontrar estudios que muestren, por ejemplo, que el $51,7 \%$ de la muestra se ubique en el estadio de acción a los seis meses de tratamiento (30). Para el paciente es el momento de modificar conductas, el momento esperado donde se han puesto las expectativas de cambio. En general creen en su propia autonomía para cambiar sus vidas, utilizan con éxito técnicas como el contracondicionamiento y el control de estímulos, y confían en el apoyo y la compresión de familiares y amigos (31).

En cuanto a los Procesos de Cambio, los que aumentan a los seis meses de tratamiento son: la liberación social, la reevaluación ambiental, el contracondicionamiento, el control de estímulos y las relaciones de ayuda. 
Encontramos otros estudios que nos recomiendan diferentes aspectos por ejemplo, al inicio de la terapia, cuando el paciente suele estar en la fase de contemplación, es cuando se requiere intensificar de forma importante el aumento de la concienciación, el relieve dramático y la reevaluación ambiental (32). También sabemos que a mayor antigüedad en el consumo y más años de abuso de la sustancia se produce mayor deterioro psicosocial y menor utilización de los recursos de apoyo (relaciones de ayuda). Un mayor historial de abuso provoca mayores dificultades para evitar situaciones de riesgo de consumo y un mayor déficit en los mecanismos de control de impulsos (33).

En el presente trabajo destacamos sobre todo el aumento del control de estímulos y el contracondicionamiento y, según Trujols y Tejero, 1994 (34), serían precisamente el control de estímulos y el contracondicionamiento los procesos más determinantes en el mantenimiento de la abstinencia y la prevención de recaídas.

A continuación pasamos a exponer los cinco procesos de cambio que aumen$\tan$ a los seis meses de tratamiento.

Contracondicionamiento. El usuario genera actividades alternativas a las conductas que son un problema, por ejemplo, hacer deporte. En primer lugar ponemos de relieve que el aumento de la variable contracondicionamiento no se debe exclusivamente al efecto de la psicoterapia, recalcamos que esta sería por añadidura una consecuencia debida al trabajo educativo. El resultado de organizar diferentes actividades de ocio y tiempo libre en una Comunidad Terapéutica motiva a los usuarios a seguir realizando esas actividades incluso en mayor cantidad y también a desarrollar actividades que nunca habían hecho (35). Según un resumen de los estudios hechos en Proyecto Hombre, en la mayoría de los casos, se da una mejoría en la relación familiar, en la satisfacción en el trabajo y con el uso del tiempo libre. Además se reduce drásticamente la obtención de dinero por fuentes ilegales. [...] De ahí que se pueda hablar más de adquisición de estilos de vida que no de simple cesación del consumo para el tratamiento de drogodependencias (36).

Control de Estímulos. No se trata de irse a vivir a otra ciudad, no se trata de evitar el problema. Se trata de modificar el ambiente con la intención de ganar confianza y esto, dadas las evidencias, sí se consigue. En cuanto al control de estímulos nuevamente la parte psico-educativa sería la mayor responsable de este aumento, por ejemplo en los diferentes talleres impartidos se aborda la recaída, cómo afrontar los estados de ansiedad, etcétera, aunque no podemos obviar que también estas temáticas son tratadas en los grupos de seguimiento terapéutico, puesto que muchos medios de los que se autoaplican los usuarios para cumplir sus objetivos tienen que ver con el control de estímulos.

Liberación Social. En cuanto al aumento significativo de la liberación social (apostar por un compromiso y responsabilizarse de dicho compromiso), los resultados son coherentes con el método y la terapia aplicada. Hay grupos de te- 
ORIGINALES Y REVISIONES

rapia donde el usuario puede expresar lo que siente, pero también se pide que las personas se responsabilicen de lo que dicen y proponen, y esto resulta de máxima importancia y prioridad en la terapia. Que un paciente llegue a la consulta pidiendo una receta farmacológica o psicológica que le dé una solución más o menos inmediata a su problema, está dentro de los lógicos parámetros de la terapia. También es frecuente llegar a la consulta afirmando que no se es conocedor de lo subjetivo de la queja. Pero lo diferente hoy en día, y con lo que nos encontramos con más frecuencia, es que el paciente no se suele sentir responsable de lo que le ocurre en relación a su malestar (37). El dolor emocional es algo que se externaliza en otros, se atribuye por ejemplo a la sociedad, al sistema, a la familia, al secreto alcoholismo del abuelo, a la educación, a los malos profesores, ¡a la pareja!, y cada día con más frecuencia, a la biología y la genética. "Ese hurto de la responsabilidad, ese empeño en disociar el pathos y el ethos de los antiguos, culminó en un nihilismo terapéutico, tanto más recalcitrante, cuanto que se privó al propio sujeto de hacerse cargo de inventar alguna solución para su desdicha" (38). En terapia es fundamental responsabilizarse de lo bueno y de lo malo, uno empieza a dejar de ser víctima cuando comienza a responsabilizarse y como consecuencia empieza a generar control, competencia y a crear alternativas. Asumir el compromiso "responsabilizarse" se ha considerado desde siempre un factor de primer orden a la hora de valorar el éxito del proceso terapéutico.

Re-evaluación Ambiental. En cuanto a la reevaluación ambiental, la persona valora qué consecuencias y beneficios acarrea el consumo de drogas y el efecto de cambiar, no solo en él mismo sino también en las relaciones interpersonales, en sus amigos y familiares. En definitiva, valora más cómo afecta su problema a los demás. De igual forma este sería otro efecto claro de la terapia ya que desde el primer momento se pide la colaboración de las figuras de apego, tanto de familiares como de amigos.

Relaciones de ayuda. El usuario desarrolla las relaciones personales que puedan ser de ayuda en su proceso de recuperación; intenta mejorar los apoyos sociales (familiares, amigos) que pueden facilitar su cambio. Esto implica que también mejoran las habilidades para mantener, recuperar o iniciar estos apoyos. Hay que subrayar que en la CT se entrenan continuamente estas habilidades dado que están en un ambiente de interacción con los demás donde los usuarios se necesitan recíprocamente.

\section{Cuestionario de Agresión ( $A Q)$}

En relación a la agresividad vemos que la variable hostilidad aumenta considerablemente. Esto lo podemos interpretar de varias formas, la primera explicación a esta evidencia es un efecto del tratamiento, puesto que aprenden a sacar fuera de ellos el conflicto. En el momento actual de tratamiento el usuario ya no se agrade a sí mismo; es decir la agresividad que supone el hacerse daño con las drogas es 
"canalizada" o dirigida de otra forma. La escala de agresividad física es un buen pronosticador de un mayor número de comportamientos agresivos (39) y vemos como en nuestro estudio la agresividad física no aumenta. Según Felsen y Hill 1999 (40), las puntuaciones altas en la escala de hostilidad están asociadas de forma significativa con un mayor nivel de ira en respuesta al maltrato personal. Esto quiere decir que a los seis meses de tratamiento los pacientes aumentan la hostilidad en respuesta al maltrato personal, es decir, un efecto de la terapia es aprender a defenderse y protegerse de la agresión externa, del maltrato ajeno (41). Pero además de las teorías anteriormente expuestas, debemos remitirnos al resultado de los ítems que se aplican a la variable "hotilidad" en el cuestionario AQ. En este sentido la explicación más lógica a este resultado es que el paciente es más consciente de su situación y suele sentir que se ha "malgastado". Se debe apuntar que la hostilidad no es lo mismo que la agresividad física o verbal, es una respuesta que en principio no debe calificarse como negativa sino más bien como una respuesta adaptativa al momento de cambio y a la fase de acción que está viviendo el paciente en ese momento, y es un resultado esperado por los terapeutas y coherente con el momento del proceso.

\section{Conclusiones}

Como hemos comprobado en el estudio, se cumplen todos los requisitos para sostener que se dan cambios significativos positivos en las variables estudiadas a causa del efecto de seis meses de tratamiento en la Comunidad Terapéutica. Por ello destacamos las siguientes conclusiones:

Las evidencias extraídas de los diferentes análisis son, entre otras, que la terapia se enfoca a las dificultades de la persona y no únicamente a los pros y contras de consumir sustancias. De este modo los pros y contras de dejar las drogas, así como las drogas en general, cada vez tendrán mucha menos relevancia, es decir, el motivo inicial por el que llegó a pedir tratamiento tiene menos importancia.

Una de las conclusiones más significativas clínicamente es que a los seis meses de tratamiento aumenta significativamente la variable autoeficacia, que $\sin$ duda tiene que ver con el éxito terapeutico.

En cuanto a los estadios de Prochaska y DiClemente, vemos que a los seis meses la única fase que aumenta es la acción, bajando las demás. Este dato nos indica que se acompaña correctamente a los usuarios a evolucionar y pasar de estados iniciales precontemplativos a la fase de acción.

En cuanto a los procesos de cambio, los que aumentan a los seis meses son:

a) contracondicionamiento: el usuario genera respuestas alternativas (tanto 
ORIGINALES Y REVISIONES

a nivel cognitivo, afectivo o conductual) a los estímulos condicionados generados por las drogas y por la conducta adictiva. La terapia ayuda a desarrollar actividades que son alternativas conductuales a la conducta problema.

b) control de estímulos: otra de las consecuencias de la terapia es que el usuario aprende a modificar el ambiente en la medida que sea posible. Y sabe cómo controlar los estímulos asociados al problema o evita las situaciones que él mismo identifica de riesgo.

c) liberación social: en cuanto al aumento de la liberación social (apostar por un compromiso y responsabilizarse del mismo) los resultados son coherentes con el método y la terapia aplicada a los seis meses de tratamiento. Asumir el compromiso "responsabilizarse", lo consideramos un factor de primer orden a la hora de valorar el éxito en el proceso terapéutico y así lo verifica este estudio.

d) reevaluación ambiental: en cuanto a la reevaluación ambiental, la persona valora qué efectos acarrea el consumo de drogas no solo sobre sí mismo, sino en las relaciones interpersonales, es decir, cómo afecta a sus amigos y familiares.

e) relaciones de ayuda: el usuario desarrolla las relaciones (familiares, amigos) y apoyos sociales que puedan ser de ayuda en su proceso de recuperación y facilitar el cambio. Tenemos que destacar en este punto que el tratamiento en la Comunidad Terapéutica es fundamentalmente una dinámica de autoayuada, el usuario aprende a establecer relaciones de ayuda de forma constante y en interacción con los demás, en un clima de ayuda mutua. Esto implica que también mejoran las habilidades para mantener, recuperar o iniciar estos apoyos.

En relación al aumento de la hostilidad. Además de las teorías anteriormente expuestas, debemos remitirnos al resultado de los ítems que se aplican a la variable "hotilidad" en el cuestionario AQ. En este sentido la explicación más lógica a este resultado es que el paciente es más consciente de su situación personal.

\section{BIBLIOGRAFÍA:}

(1) Marina P. ¿Qué nos han enseñado los estudios de seguimiento de toxicómanos? Adicciones. 1999; Vol 1, n³: 237-241

(2) Bedate J, Romero J. Evaluación de 84 heroinómanos un año después del tratamiento hospitalario del síndrome de abstinencia. 1985; XIII Jornadas de Sociodrogalcohol. Palma de Mallorca.

(3) Melgajero M, Sanahuja P, Masferrer J. Estudio de seguimiento en población heroinómana. Fons Informatiu 1986; 1:1-38.

(4) Sánchez-Carbonell X, Brigos B, Camí J. Evolución de una muestra de heroinómanos dos años después del inicio del tratamiento (proyecto EMETYST). Med Clín, 1989; Vol. 92, No4:135-139.

(5) Fernández, J, Secades, R. Estudio comparativo de la eficacia de los programas de tratamiento de Proyecto Hombre de Asturias, Galicia y Baleares. Ed. Madrid: Asociación Proyecto Hombre. Subvenciona: Ministerio del Interior. Delegación del Gobierno para el Plan Nacional sobre Drogas, 2001. 
(6) Goñi J. Evaluación de la Eficacia de la Comunidad Terapéutica de Proyecto Hombre de Navarra. Tesis Doctoral. Departamento de Psicología y Pedagogía Universidad Pública de Navarra, 2005.

(7) García E, Roldán G, Comas D. Un estudio de seguimiento de la Comunidad Terapéutica Haize-Gain. Ed. Asociación Guipuzcoana de Investigación y Prevención del Abuso de las Drogas. AGIPAD, 2010.

(8) DSM-IV. Manual diagnóstico y estadístico de los trastornos mentales. Barcelona: Masson, 1998.

(9) Bobes J, González M, Sáiz P, Bousoño M. Índice europeo de severidad de la adicción: EuropASI. Versión española. Actas de la IV Reunión Interregional de Psiquiatría, 1996; 201-218.

(10) Derogatis LR. SCL-90-R. Cuestionario de 90 síntomas [SCL-90-R. Ninety Symptoms. Questionnaire.] Madrid: TEA Ediciones, 1975.

(11) Loranger AW, Sartorius N, Andreoli A, Berger P, Buchheim, P, Channabasavanna SM, Coid B, Dahal A, Diekstra RF, Ferguson B, Jacobsberg L B, Mombour W, Pull C, Reiger D. The world Health Organization/Alcohol, Drug Abuse and Mental Health Administration International Pilot Study of Personality Disorders. Arch Gen Psychiat 1994; 51: 215-224. Adaptado en España por: López-Ibor JJ, Pérez A, Rubio V. Examen internacional de los Trastornos de la personalidad. (IPDE). Madrid: Meditor, 1996.

(12) McCnnaughy EA, DiClemente CC, Prochaska JO, Velicer WE. Stages of change in psychotherapy: A follow-up report. Psychother-Theor Res 1989;20: 368-375.

(13) Prochaska J, Norcross JC, Diclemente CC, Changing for good. The revolutionary program that explains the six stages of change and teaches you how to free yourself from bad habits. New York: William Morrow and Company, 1994.

(14) Trujols J, Tejero A. Evaluación cognitivo conductual y psicodiagnóstica del trastorno por dependencia de opiáceos. En: Becoña E, Rodríguez A, Salazar I, (eds.), Drogodependencias. I Introducción. (p.163-208). Santiago de Compostela: Servicio de publicaciones e intercambio científico de la Universidad de Santiago de Compostela, 1994.

(15) Prochaska JO, Prochaska JM. Modelo transteórico del cambio de conductas adictivas. En: Casas, EM. Gossop (eds.), Recaída y prevención de recaídas. Tratamientos psicológicos en drogodependencias (p. 56-85). Barcelona: Ediciones de neurociencias, 1993.

(16) Prochaska J, Norcross JC, Diclemente CC. Changing for good. The revolutionary program that explains the six stages of change and teaches you how to free yourself from bad habits. New York: William Morrow and Company, 1994.

(17) Trujols J. Tejero A, Evaluación cognitivo conductual y psicodiagnóstica del trastorno por dependencia de opiáceos. En: Becoña E, Rodríguez A, Salazar I, (eds.), Drogodependencias. I Introducción. (p.163-208). Santiago de Compostela: Servicio de publicaciones e intercambio científico de la Universidad de Santiago de Compostela, 1994.

(18) Velicer WF, DiClemente CC, Rossí JS, Prochaska JO. Relapse situations and self-efficacy: an integrative model. Addict Behav 1990;15:271-283.

(19) Buss AH, Perry M. The aggression questionnaire. J Pers Soc Psychol, 1992; 63: 452-459.

(20) Graña JL, Nuñoz JJ, Navas E. Características psicopatológicas, motivacionales y de personalidad en drogodependencias en tratamiento de la Comunidad de Madrid. Madrid: Madrid Salud. Agencia Antidroga, 2007.

(21) Emrick C. Alcoholics Anonymous: Affiliation processes and effectiveness as treatment. Alcohol Clin Exp Res 1978; 11: 416-23. 
(22) Deben O, Fernández MC, López-Goñi JJ, Illescas C, Santos JR. La Evaluación de la eficacia en Proyecto Hombre. En: AAVV Desafíos y avances en la prevención y el tratamiento de las drogodependencias. Madrid: Asociación Proyecto Hombre, 2004.

(23) Miller R, Rollnick S. La entrevista motivacional. Barcelona: Paidós, 1999.

(24) Álvarez JM, Esteban R, Sauvagnat F. Fundamentos de psicopatología psicoanalítica. Madrid: Síntesis, 2004.

(25) Oiumette P, Finney JW, Moss RH. Twelve-step and cognitive - behavioural treatment for substance abuse: a comparison of treatment effectiveness. Consult Psychol, 1977; 65: 230-40.

(26) Burling TA, Reilly PM, Moltzen JO, Ziff DC. Self-efficacy and relapse among impatient drug and alcohol abuses: A predictor outcome. J Stud Alcohol , 1989; 50: 354-360.

(27) Gossop M, Green L, Phillips G, Bradley B. Factors predicting outcome among opiate addicts after treatment. Brit J Clin Psychol 1990; 29: 209-216.

(28) DiClemente CC. Self-efficacy and the addictive behaviours. J Soc Clin Psychol 1986; 4: $302-315$.

(29) DiClemente CC. Self-efficacy and the addictive behaviours. J Soc Clin Psychol 1986; 4: 302-315.

(30) Santos P. Estadios de cambio en drogodependientes en tratamiento ambulatorio. Adicciones 2001: Vol. 13. $\mathrm{N}^{\mathrm{o}} 2 ; 147-152$.

(31) Martín F. Redes y programas para el tratamiento de las drogodependencias. Estudios de Juventud, 1997; 40: 79-93.

(32) Días J. El modelo de los estadios de cambio en la recuperación de las conductas adictivas. Escritos de psicología, 2001; 5: 21-35

(33) Sánchez-Hervás E, Grandolí T, Bou M. Proceso de cambio en conductas adictivas: Influencia de variables psicopatológicas y de consumo. Adicciones. 2002; Vol 14. No 3: 337-344.

(34) Tejero A, Trujols J. Evaluación cognitivo conductual y psicodiagnóstica del trastorno por dependencia de opiáceos. En: Becoña E, Rodríguez A, Salazar I, (eds.), Drogodependencias. I Introducción. (p.163-208). Santiago de Compostela: Servicio de publicaciones e intercambio científico de la Universidad de Santiago de Compostela, 1994.

(35) Pérez F, Lara F, González M. Ocio y tiempo libre en drogodependientes. Infad. Revista de Psicología. International Journal of Developmental and Educational Psychology, 2009; 1: 93-102

(36) Deben O, Fernández Ma C, López-Goñi JJ, Illescas C, Santos JR. La Evaluación de la eficacia en Proyecto Hombre. En AAVV Desafíos y avances en la prevención y el tratamiento de las drogodependencias. Madrid: Asociación Proyecto Hombre, 2004.

(37) Pérez F, Martín I. Nuevas Adicciones ¿Adicciones Nuevas? Guadalajara: Ediciones Intermedio, 2007.

(38) Álvarez JM. La angustia entre goce y deseo. Análisis, 2008; 16: 29-43.

(39) Bushman BJ, Well GL. Trait aggressiveness and hockey penalties: Predicting hot tempers on the ice. J Appl Phycol 1998; 83: 969-974.

(40) Felsen G, Hill V. Aggression Questionnaire hostility scale predicts anger in response to mistreatment. Behav Res Ther 1999; 37: 87-97.

(41) Felsen G, Hill V. Aggression Questionnaire hostility scale predicts anger in response to mistreatment. Behav Res Ther 1999; 37: 87-97. 